\title{
October 2016 Critical Care Case of the Month
}

\author{
Stephanie Fountain, MD \\ Banner University Medical Center Phoenix \\ Phoenix, AZ USA
}

A 27-year-old Caucasian man with past medical history of opioid abuse (reportedly sober for 10 years on buprenorphine), post traumatic stress disorder, depression and anxiety presented to the emergency department complaining of dysarthria after taking diphenhydramine and meclizine in addition to his prescribed trazodone and buprenorphine to try to sleep. He was discharged to home after his symptoms appeared to improve with intravenous fluid.

He returned to the emergency department the following afternoon with worsening dysarthria, dysphagia, and subjective weakness. The patient was non toxic appearing, afebrile, vital signs were stable and his strength was reported as 5/5. Computed tomography of his head did not show any evidence of acute intracranial abnormality. Given his ongoing complaints, he was admitted for observation to the general medicine wards.

That night a rapid response was initiated when the nurse found the patient to be unresponsive, but spontaneously breathing. The patient's clinical status did not change with naloxone administration. An arterial blood gas obtained demonstrated a profound respiratory acidosis with a pH of 7.02 and a pCO2 of 92 . He was emergently intubated. A chest x-ray was performed (Figure 1).

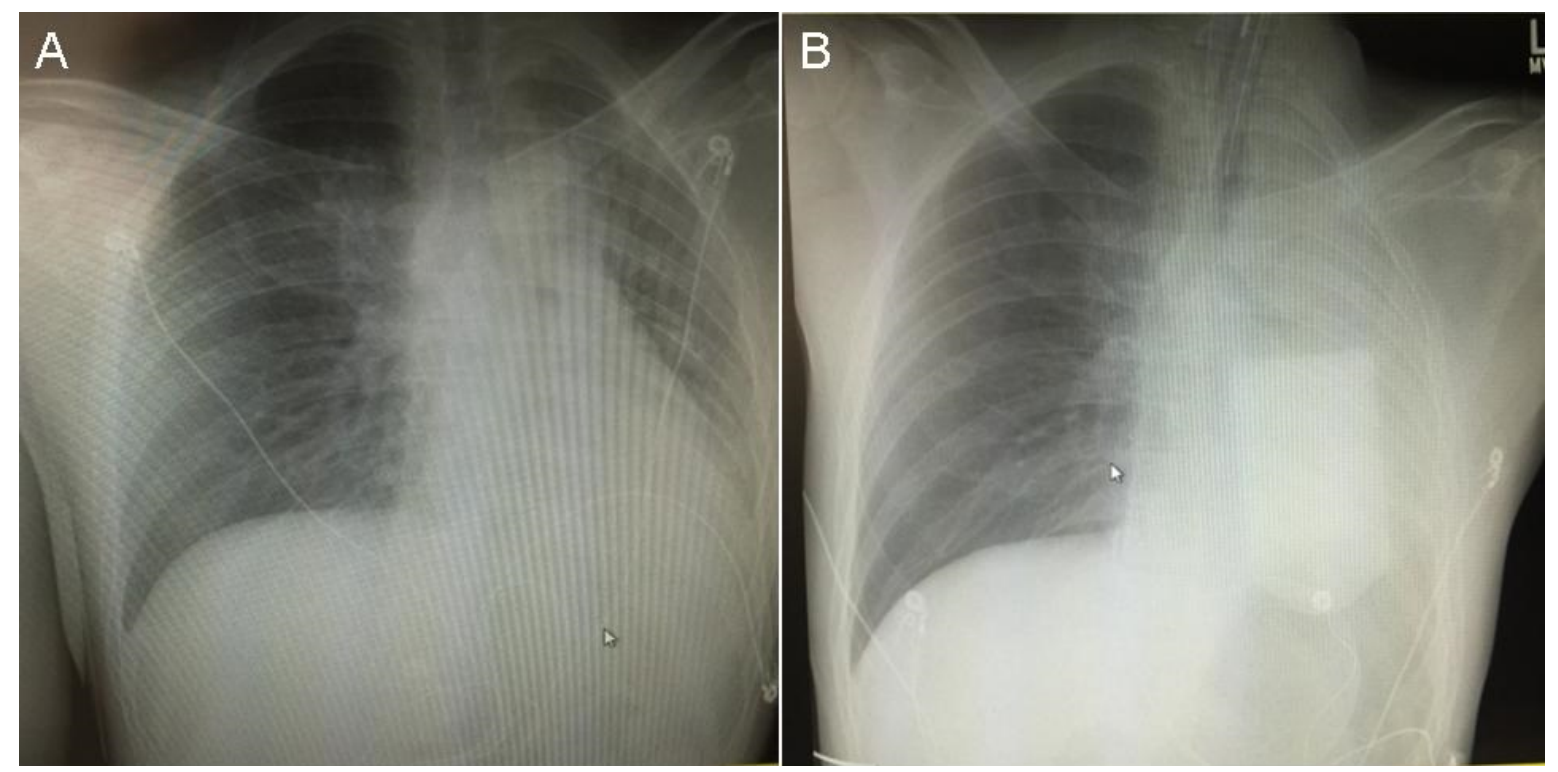

Figure 1. Panel A: admission portable chest $\mathrm{x}$-ray. Panel B: chest -ray immediately after intubation. 
Which of the following are present on his chest X-ray?

1. Left lung atelectasis

2. Left pleural effusion

3. Right mainstem intubation

4. 1 and 3

5. All of the above 


\section{Correct!}

\section{Left lung atelectasis}

His chest $x$-ray shows rapidly increasing density in the left chest with left lung volume loss. The tip of the endotracheal tube is visible in the trachea. Pleural effusion can cause increased density in the chest but is not associated with volume loss unless there is accompanying atelectasis. His CXR about 12 hours after his intubation is shown in Figure 2.

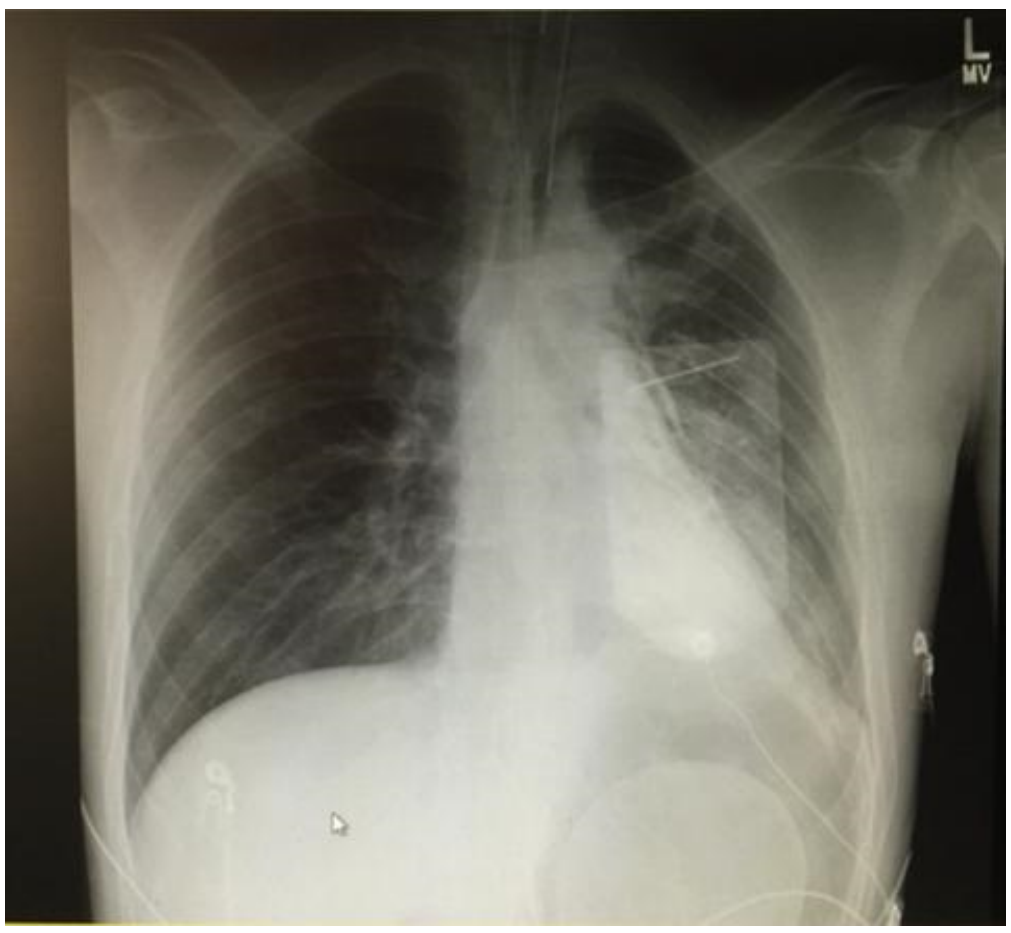

Figure 2. Chest $x$-ray the next morning after being on volume control ventilator settings overnight. The rapid clearing of the density on the right is consistent with atelectasis.

After transfer to the ICU, his neurologic exam was significant for inability to open his eyes bilaterally, blurred vision (after manual opening of his eyelids), dilated but reactive pupils $(8 \mathrm{~mm})$ and symmetric, bilateral, proximal muscle weakness with intact sensation but absent reflexes. He was able to move all 4 extremities spontaneously and could communicate by writing.

Which diagnosis is least likely given the presentation?

1. Amyotrophic lateral sclerosis

2. Botulism

3. Guillain-Barré syndrome

4. Lambert Eaton myasthenic syndrome

5. Myasthenia gravis 


\section{Correct! \\ 1. Amyotrophic lateral sclerosis}

Amyotrophic lateral sclerosis (ALS) is characterized by combined upper motor neuron and lower motor neuron signs. While this patient's ascending symmetric weakness could be consistent with lower motor neuron findings, he lacks any upper motor neuron findings like hyperreflexia, spasticity, or fasciculations.

Magnetic resonance imaging of his brain was unremarkable as was analysis of his cerebral spinal fluid. A small $1 \times 1 \mathrm{~cm}$ mildly indurated area on his left forearm was found (Figure 3). Upon further questioning without family in the room, patient admitted to recent subcutaneous injection of black tar heroin.
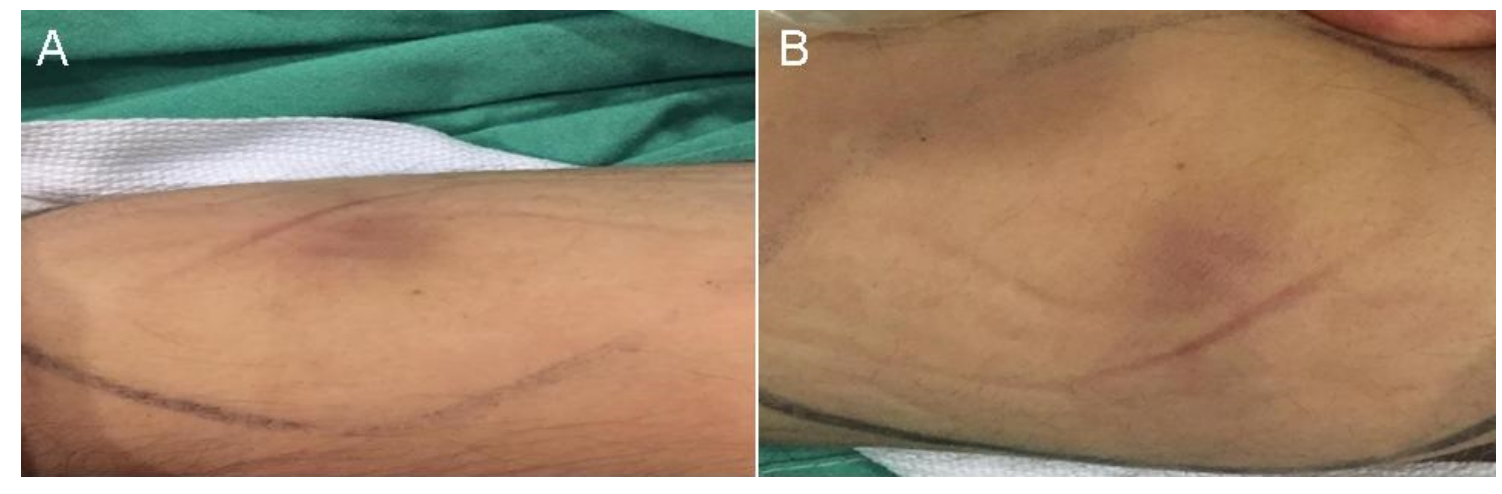

Figure 3. Panel A: Photograph of the area of induration on the left forearm. Panel B: close up of the area.

Given his ongoing drug use, which diagnosis is most likely?

1. Amyotrophic lateral sclerosis

2. Botulism

3. Guillain-Barré syndrome

4. Lambert Eaton myasthenic syndrome

5. Myasthenia gravis 


\section{Correct! \\ 2. Botulism}

Botulism is a rare and potentially life threatening neuroparalytic condition mediated by a neurotoxin produced by the bacterium Clostridium botulinum (1). There are six forms of botulism distinguished by the route of infection: foodborne, infant, adult enteric, wound, iatrogenic and inhalation. According to the Centers for Disease Control and Prevention (CDC), there are fewer than 5 reported cases of wound botulism in the US per year (2). Despite clostridium being ubiquitous, these tend to occur only in patients who subcutaneously inject black tar heroin, and may be an under recognized cause of death in presumed opioid over dose $(3,4)$.

When $C$. botulinum spores are injected into a wound, the devitalized tissue provides the ideal medium for anaerobic growth. Seven serotypes of toxin can be produced, of which toxins $A, B, E$ and $F$ can cause human disease. Paralysis occurs through toxinmediated blockade of neuromuscular transmission in cholinergic nerve fibers.

It is unclear why black tar heroin injection subcutaneously or intramuscularly ("skin popping") vs. intravenous injection is associated with wound botulism. Black tar heroin is becoming more prevalent in the US as it is a relatively crude and unrefined opiate which does not require the complex equipment, expensive chemicals and lengthy steps required to produce pure heroin or "china white" (Figure 4).

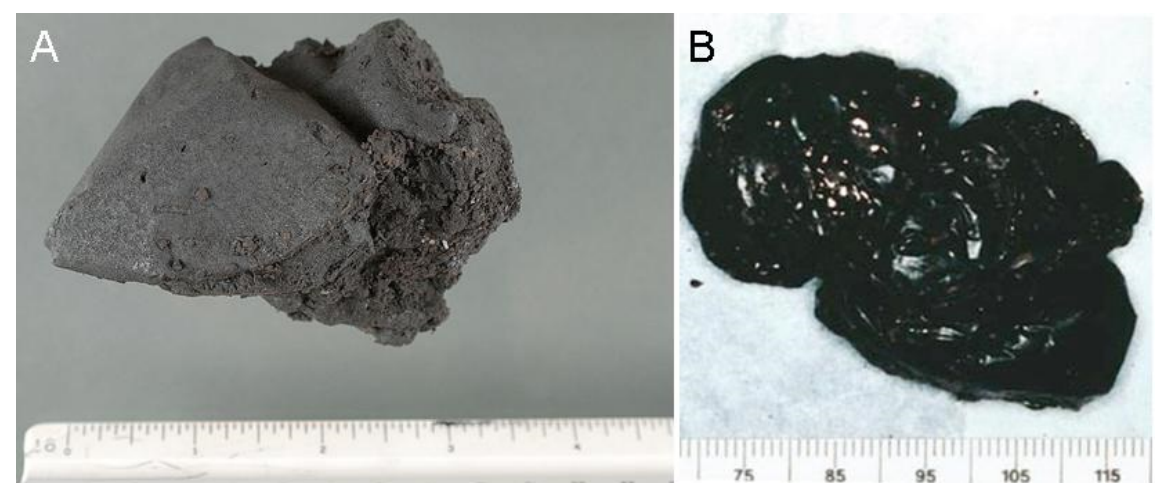

Figure 4. Photographs of black heroin.

Botulism was suspected given the clinical picture and active black tar heroin use. The anti-toxin was acquired from the CDC and administered.

Diagnosis was later confirmed by murine bioassay of serum, wound and stool and anaerobic wound culture. This case met CDC criteria for report of confirmed wound botulism (Table 1).

Table 1. CDC Case Criteria for Would Botulism Clinical Description 
An illness resulting from toxin produced by Clostridium botulinum that has infected a wound. Common symptoms are diplopia, blurred vision, and bulbar weakness. Symmetric paralysis may progress rapidly.

\section{Laboratory Criteria for Diagnosis}

- Detection of botulinum toxin in serum, OR

- Isolation of Clostridium botulinum from wound

Case Classification

\section{Probable}

A clinically compatible case in a patient who has no suspected exposure to contaminated food and who has either a history of a fresh, contaminated wound during the 2 weeks before onset of symptoms, or a history of injection drug use within the 2 weeks before onset of symptoms. Confirmed

A clinically compatible case that is laboratory confirmed in a patient who has no suspected exposure to contaminated food and who has a history of a fresh, contaminated wound during the 2 weeks before onset of symptoms, or a history of injection drug use within the 2 weeks before onset of symptoms.

Although rare, botulism is important to keep in the differential diagnosis, especially in patients who inject black tar heroin subcutaneously as early recognition, prompt antitoxin administration, and complete source control are essential in preventing poor outcomes in an otherwise reversible neurological condition.

\section{References}

1. Centers for Disease Control and Prevention. Botulism. Available at: https://www.cdc.gov/botulism/ (accessed 9/12/16).

2. Centers for Disease Control and Prevention. Botulism / c. botulinum (Clostridium botulinum) 2011 Case Definition. Available at: https://wwwn.cdc.gov/nndss/conditions/botulism/case-definition/2011/ (accessed 9/12/16).

3. Passaro DJ, Werner SB, McGee J, Mac Kenzie WR, Vugia DJ. Wound botulism associated with black tar heroin among injecting drug users. JAMA. $1998 \mathrm{Mar}$ 18;279(11):859-63. [CrossRef] [PubMed]

4. Centers for Disease Control and Prevention. Wound botulism among black tar heroin users--Washington, 2003. MMWR Morb Mortal Wkly Rep. 2003 Sep 19;52(37):885-6. [PubMed] 